\title{
Bearings-Only Tracking of Manoeuvring Targets Using Multiple Model Variable Rate Particle Filter with Differential Evolution
}

\author{
Ghasem Saeidi, M. R. Moniri
}

Department of Communication Engineering, Islamic Azad University, Shahr-e-Rey Branch, Iran

\begin{abstract}
In standard target tracking, algorithms assume synchronous and identical sampling rate for measurement and state processes. Contrary to these methods particle filter is proposed with variable rate. These filters use a restricted number of states, and a Gamma distribution is applied at state arrival time so that the maneuvering targets could be tracked. Although this structure is capable of tracking a wide range of targets motion features using linear, curvilinear motion dynamics, it suffers from a basic weak point. It cannot estimate the position of targets in high maneuvering regions. Thus, multiple model variable rate particle filter (MM-VRPF) is utilized to overcome this shortage using various dynamic models. A weak point of a particle filter is a phenomenon called degeneracy that even exists in MM-VRPF structure. In this study, differential evolution method, is exploited to improve the mentioned method and a novel structure called multiple model variable rate particle filter with differential evolution (MM-VRPF with DE) is introduced. The simulation results of a bearing only tracking achieved from a maneuvering target, revealed that the proposed structure has better performance while it maintains advantages of variable rate structure.
\end{abstract}

Key words: Target tracking, Multiple model variable rate particle filter, Differential evolution
This article is is licensed under a Creative Commons AttributionNonCommercial 4.0 International License.

Attribution-NonCommercial (CC BY-NC) license lets others remix, tweak, and build upon work non-commercially, and although the new works must also acknowledge \& be noncommercial.

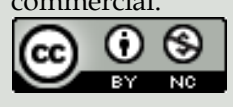

Source of Support: Nil

Conflict of Interest: Declared

\section{INTRODUCTION}

General speaking tracking is referred to obtaining kinematic parameters of the target (such as location, velocity, acceleration) during a time interval based on noisy observations. During the last decade tracking maneuvering targets have experienced increasing progress and has attracted great attention owing to the development of numerical techniques (Jilkov, 2003). Estimation in nonlinear systems is a prominent issue in many 
applications. Bayesian field is one of the most popular estimation techniques. From its perspective, the objective is to estimate a stochastic process based on noisy observations. In this framework state space equations are common. The goal is to estimate the state of a dynamic system based on noisy observations that are a function of system state. In Bayesian filter, it is desired to estimate posterior probability density function. Knowing the probability density, an optimized estimation of states might be achieved, and it could be calculated proportionate to every criterion function. Since this filter does not have a closed solution, different methods have been proposed for its implementation in accordance with process and measurement model. With this regard for limited linear dynamic systems grid, based filters are utilized Fox (2003), Boers (1999). Furthermore in case of a nonlinear system and Gaussian noise Extended Kalman Filter (EKF) is exploited Ristic (2004), Simon (2006). Increase in nonlinearity of the system, the estimation results are distorted, and the posterior probability function violates Gaussian state and destroys the estimations Ristic, (2004), Arulampalam (2002). Another practical solution for implementation of Bayesian filters is using nonparametric methods among that the most important one is the particle filter (Gordon, 1993). In the above-mentioned filter posterior probability density function is estimated by a set consisting of weighted a particles Ristic, (2004), Arulampalam (2002).

In standard methods for target tracking and particularly in particle filter, the state sampling rate is determined proportionate to measurement rate. A modern and economic approach is utilizing variable rate particle filter (VRPF) where state arrival times (new states) are modeled as pseudo-Markovian random process. Although this structure would be able to track different features of motion using linear, curvilinear motion dynamic model, it is not capable of providing a precise estimation in regions with high maneuver. To address this problem, a structure with multiple models might be employed that models, target motion dynamics using a set of models and it can switch between these models. The modified structure is called multiple mode variable rate particle filter (MMVRPF). Using this method continuous certain process proposed in Godsill (2007), Ulker (2012) will be maintained; meanwhile, they would be adapted to variable rate structure with multiple models, so that the tracking operation is improved.

The most essential weak point that must be taken into considerations in particle filters is the degeneracy phenomenon that results from an increase in variance of weights (Doucet, 2000). In practice, it has been observed that most of the samples have normalized weight close to zero after a short time, and only one sample has a large weight. So the weights of some samples are calculated whereas they have a negligible effect on the final estimation that is a waste of power. To address this issue resampling is utilized. In resampling stage, weighted samples at the end of a step, are sampled $\mathrm{N}$ times. The chance of each sample for being selected depends on its weight. As a result, in this step samples with greater weights are copied several times and samples with smaller weights would be eliminated. At the end of this step a non-weighted estimation of joint posterior distribution is achieved. Numerous algorithms have been proposed for resampling; in (Doucand, 2005) a good comparison is presented. Resampling method improves degeneracy (Gordon, 1993); however, it has a crucial weakness called sample impoverishment. It is due to repeat of samples with large weight. It causes all samples to have the same history after a specific time step. In this paper differential evolution optimization algorithms are utilized to mitigate degeneracy an a new set of filters are introduced; multiple model variable rate particle filter with differential evolution (MM-VRPF with DE). In this algorithm particles are optimized using differential evolution algorithm and they are combined with a random set obtained by probability distribution in variable rate structure, so that better solution is derived. 
The simulation results illustrated that proposed structure increases efficiency and precision in path estimation compared to MM-VRPF.

\section{Variable Rate Particle Filter and Modeling Target Motion}

This paper focuses on improving tracking operation and increasing estimation precision in MM-VRPF. In order to understand other sections here, a brief review on the structure of, variable rate particle filter is provided. More details might be found in Ulker (2012), Punch (2012).

In standard constant rate stat-space models a state variable $x_{t}$ is defined that evolves during time with $t$ index. The generic model is considered between time $\{0$ and $T\}$. variable state sequence follows a Markovian process and they are generated based on density function shown in equation (1) Godsill (2007), Ulker (2012).

$x_{k} \sim p\left(x_{k} \mid x_{k-1}\right), \quad \tau_{k}>\tau_{k-1}$

Where $x_{k}$ is State with variable rate is defined in the form of $x_{k}=\left(\tau_{k}, \theta_{k}\right), \mathrm{k}$ is a discrete index and $\tau_{k}$ and $\theta_{k}$ respectively denote new state arrival in witch state, and a vector of target parameters.

In a variable rate model state assignment is not synchronous with observations. Thus, the optimum solution is when state positions (new states) are dependent on a probability function. As a matter of fact, it is assumed that an observation is independent of all data points except neighboring points. Similarity probability function for consecutive values of $\mathrm{t}$ could be defined as equation (2) (Ulker, 2012):

$p\left(\mathrm{y}_{t} \mid x_{0: \infty}\right)=p\left(\mathrm{y}_{t} \mid x_{N_{t}}\right)$

Where $x_{N_{t}}=\left\{x_{k} ; \mathrm{k} \in N_{t}\left(x_{0: \infty}\right)\right\}$ and $y_{t}$ is an observation.

It is noteworthy that $N_{t}$ includes all states close to observations at times t. A process in the form of $\widehat{\omega}_{t}=f_{t}\left(\mathrm{x}_{N_{t}}\right)$ is defined that might be utilized for calculating probability function. It is assumed that the largest and smallest elements of neighboring set are $N_{t}^{+}$ and $N_{t}^{-}$, respectively. Finally, common density of observations and states could be demonstrated as shown in equation (3) according to Markovian assumptions (Ulker, 2012).

$$
p\left(x_{0: k}, z_{0: T}\right)=p\left(x_{0}\right) \prod_{l=1}^{k} p\left(x_{l} \mid x_{l-1}\right) \prod_{t=0}^{T} P\left(\mathrm{y}_{t} \mid x_{N_{t}}\right), \quad K \geq N^{+}{ }_{T}
$$

Where $K \geq N_{T}^{+}$guarantees a complete neighborhood for calculating density observed at the end of $\mathrm{T}$ time index.

Defining $z_{0: t}=\left(z_{0}, \ldots, z_{t}\right)$ as observations and $x_{0: \mathrm{N}^{+}}=\left(x_{0}, \ldots, x_{{N^{+}}^{+}}\right)$as desired target states (that is always a random variable), it can be said that at each time step $t$, VRPF structure will result in an estimation of optimized filtering distribution. It is denoted (as shown by equation (2) (Ulker, 2012)) in the form of a combination of $N_{p}$ "multi dimensional" Dirac delta each of that illustrates a particle.

$p\left(\mathrm{X}_{0: \mathrm{N}^{+}}, N^{+}{ }_{t} \mid z_{0: t}\right) \approx \sum_{i=1}^{N_{d}} \omega_{t}^{i} \sigma\left(x_{0: N^{+}{ }_{t}}^{i}-x_{0: \mathrm{N}^{+}}\right)$ 
Where $\omega_{\mathrm{t}}^{\mathrm{i}}$ is the weight of its particle. The above equation calculates $w_{\mathrm{t}}^{\mathrm{i}}$ at state arrival time by performing updating operation based on equation (5) (Ulker, 2012) and $\omega_{\mathrm{t}-1}^{\mathrm{i}}$.

$\omega_{t}^{i} \propto \omega_{t-1}^{i} \frac{p\left(y_{t} \mid x_{N_{t}}^{i}\right) p\left(x_{N_{t-1}+1: \mathrm{N}^{+}}{ }_{t} \mid x_{N^{+}{ }_{t-1}}^{i}\right)}{q\left(x_{N^{+}{ }_{t-1}+1: \mathrm{N}^{+}} \mid x_{N^{+}{ }_{t-1}}^{i}, y_{0: t}\right)}$

As mentioned before in a conventional variable rate particle filter merely, one model is exploited to estimate the position of the target. According to (Godsill, 2007) a CL model would be an appropriate choice in such filter for modeling target motion.

In CL model tangent and perpendicular motions depend on the target. If consider $\mathrm{R}$ as the passed motion path and $L_{T, K}$ and $L_{P, K}$ as dynamic value of tangent motion and pitch motion, respectively, equation (6) could be derived.

$L_{P, K}=m \frac{d s}{d t} \frac{d \psi}{d t}, \quad L_{T, K}=m \frac{d^{2} s}{d t^{2}}$

Where $\psi$ are a relative angle with $l_{1}$ an axis, $\mathrm{m}$ is the mass of target and $\mathrm{t}$ lies in $\tau_{k}\left\langle t \leq \tau_{k-1}\right.$. As a result, target state vector $\theta_{k}$ is illustrated by a vector in the form of equation (7).

$\left[\begin{array}{lllll}L_{P, K} & L_{T, K} & V(\tau k) & \psi(\tau k) & z(\tau k)\end{array}\right]$

Where $\psi(\tau k)$ is the course and $V(\tau k)$ is the velocity of the target. $V(\tau k)=\left[l_{1} l_{2}\right]$ is the $K^{\text {th }}$ state vector in $l_{1}$ and $l_{2}$ coordinates.

\section{Multiple Model Variable Rate Particle Filter}

In standard VRPF method new state arrival time and target motion are configured using a united model. However, during a maneuver motion parameters and arrival, times are diverse due to the nature of targeting problem. On this basis usually state arrival times and target maneuver parameters are not estimated with a unique model. To improve the structure, a multiple model variable rate structure, is proposed. In this structure, arrival times and maneuver parameters are modeled by a model consisting of a triplet set of parameters that improves targeting operation. In this method another state variable $\left(m_{k}\right)$ is added to the state vector of VRPF. It shows dynamic motion mode and is denoted by equation (8) (Ulker, 2012).

$x_{k}=\left[\tau_{k}, \theta_{k}, m_{k}\right] \quad m_{k} \in[1, \ldots, \mathrm{j}]$

Where $\mathrm{j}$ means all states. Each targeting plan or program is used for demonstration of a set of dynamic states. Each state particularly demonstrates a specific feature of the target maneuver. In this paper we deal with a model consisting of three states so $\mathrm{j}$ is selected to be three. It is worth mentioning that increase in a number of states does not necessarily lead to improvement of filter performance. Thus, selecting the states in a "multiple model" system must be a function of desired complexity (Ulker, 2012). The desired structure is demonstrated in equation (9) (Ulker, 2012). 


$$
\begin{aligned}
p\left(x_{k} \mid x_{k-1}\right)= & p\left(\theta_{k} \mid \theta_{k-1}, \tau_{k}, \tau_{k-1}, m_{k}\right) \\
& \times p\left(m_{k} \mid m_{k-1}\right) \mathrm{p}\left(\tau_{k} \mid \tau_{k-1}, m_{k-1}\right)
\end{aligned}
$$

Where $p=\left(m_{k} \mid m_{k-1}\right)$ is the probability of state transition. It shows the probability of transition from one state to another and staying in a specific state. These probabilities are demonstrated by state transition matrix $\mathrm{p}$ as shown in equation (10) (Ulker, 2012).

$$
p=\left[\begin{array}{ccc}
p_{11} & \cdots & p_{1 r} \\
\vdots & \ddots & \vdots \\
p_{r 1} & \cdots & p_{r r}
\end{array}\right]
$$

Where $p_{h l},\{\mathrm{~h}, 1\} \in\{1, \ldots, \mathrm{r}\}$ demonstrates values of probability of transition from $h$ to $l$. Practically matrix $\mathrm{p}$ is directly determined based on desired target maneuver. In (Bloomer, 2002), some specific methods for selecting $p$ are mentioned. Moreover, some methods for online calculation of p matrix could be found in (Jilkov, 2004).

Consequently for target motion kinematic vector $\theta_{k}$ can be illustrated by $\left[\begin{array}{lllll}L_{P, K} & L_{T, K} & V(\tau k) & \psi(\tau k) & z(\tau k)\end{array}\right]$ (Ulker, 2012) where $L_{P, K}$ and $L_{T, K}$ could be denoted by equations (11) and (12) in the form of Gaussian distribution (Ulker, 2012).

$$
\begin{aligned}
& L_{P, K} \sim N\left(0, \sigma^{2}{ }_{p, n}\right) \\
& L_{T, K} \sim N\left(\mu_{T}, \sigma^{2}{ }_{p, n}\right)
\end{aligned}
$$

Furthermore, $p\left(\tau_{k} \mid \tau_{k-1}, m_{k-1}\right)$ in equation (9), is conditional to discrete variable the mode. The previous arrival time is shown by a shifted Gamma distribution as denoted by equation (13) (Ulker, 2012).

$$
\tau_{k}-\tau_{k-1}-\tau_{n} \sim G\left(\alpha_{n}, \beta_{n}\right)
$$

Where $\tau_{n}$ is the sojourn time shifting parameter and $n=m_{k-1}, m_{k-1} \in\{1, \ldots, r\}$ demonstrates model motion mode. Therefore, new state arrival time $\tau_{\mathrm{k}}^{\mathrm{i}}$ on $m_{k}$ mode and previous states arrival time $\tau_{\mathrm{k}-1}^{\mathrm{i}}$ (where $i=1, \ldots, \mathrm{N}_{P}$ and $N_{p}$ is the number of samples selected from an initial distribution) are generated according to equation (13).

Now a combination of multiple model structure with variable rate models is presented. Similar to standard structure in the multiple model variable rate structure mentioned steps are taken to estimate the state.

\section{Initial setting}

In this stage values are assigned to all particles according to a determined distribution. At time $\mathrm{t}=0, N_{p}$ samples are selected; then, selected samples are weighted based on their similarity to actual value in the form of equation (14).

$\omega_{t=0}^{i}=\frac{1}{N_{p}}, i=1, \ldots, \mathrm{N}_{P}$.

Where $\omega_{t=0}^{i}$ is particle weight at time $\mathrm{t}=0$. 
Asia Pacific Journal of Energy and Environment, Volume 2, No 2 (2015) 


\section{Propagation step}

In this step as soon as a new state arrives, $N_{p}$ samples are selected based on $\mathrm{q}($. distribution that plays the role of previous state distribution. $\mathrm{q}($.$) distribution is stated as$ shown by equation (15) (Ulker, 2012).

$q\left(x_{N_{t-1}^{+}+1: \mathrm{N}_{t}^{+}} \mid x_{N_{t-1}^{+}}^{i}, y_{o: t}\right)=p\left(x_{N_{t-1}^{+}+1: N_{t}^{+}} \mid x_{N_{t-1}^{+}}^{i}\right)$

\section{Updating the particle weights}

Updating is performed based on a simplified form of equation (5). In this equation if previous distribution $\mathrm{q}($.) is utilized, a simpler equation in the form of equation (16) is derived (Ulker, 2012) that is exploited for calculating particle weights $\omega_{t}^{i}, \quad i=1, \ldots, \mathrm{N}_{P}$.

$\omega_{t}^{i} \propto \omega_{t-1}^{i} p\left(y_{t} \mid x_{N_{t}}^{i}\right)$

Thus, $p\left(y_{t} \mid x_{N_{t}}^{i}\right)$ probability in updating operation of $\omega_{t}^{i}$ could be defined in the form of equation (17).

$$
p\left(y_{t} \mid x_{N_{t}}\right)=p\left(y_{t} \mid \widehat{\omega}_{t}\right)
$$

Finally, it could be concluded that posterior probability function $p\left(x_{\mathrm{N} t}, \mathrm{~N}_{t}^{+} \mid y_{0: t}\right)$ is estimated by associated weight vectors $\left\{y_{t}^{i}\right\}_{i=1}^{N_{p}}$ and particles $\left\{x_{\mathcal{N}_{t}}^{i}\right\}_{i=1}^{N_{p}}$. Afterwards, if $\widehat{N}_{\text {eff }}=\frac{1}{N_{p}}$ is less than default threshold value, resampling operation will be done. $\sum_{i=1}^{N_{p}}\left(w_{t}^{i}\right)^{2}$

MM-VRPF structure does not need regeneration stage whereas it is necessary for VRPF framework; thus, it considerably reduces a computational load (Ulker, 2012).

\section{DifFERENTIAL EVOLUTION}

Differential evolution (DE) is a differential algorithm proposed by Storm and Price in 1995. This algorithm uses evolution operators such as other differential algorithms; nevertheless, this method utilizes current distance and direction information to guide searching process. The most important operator in the evolutionary algorithm is a mutation operator that is utilized for generating target vector and children (Engelbrecht, 2007). In fact, differential evolution is an optimization method for "multi dimensional" functions including a population of possible solutions. High speed and simplicity are characteristics of this method. If there is no default information regarding analysis space, an initial population might be generated randomly.

In this paper standard version of DE i.e. (DE/rand/1/bin) is utilized. This method starts by setting three parameters $C_{r}, N_{p}$ and $\mathrm{F}$ that denote probability of a turn, size of population and mutation weight, respectively. According to Storm's assumption, these values are set between $\{0$ to 2$\}$. Choosing smaller values for $F$ increases a convergence speed while higher values result in more diversity in generated population. 
The above algorithm, first off, randomly generates a population in the size of $N_{p}$. Besides, this algorithm includes three stages mutation, recombination and selection. A population in the form of $p_{G}^{i}$ is considered and a vector whose length is $N_{p}$. The vector includes elements in the form of $p_{\mathrm{J}, \mathrm{G}}^{i}$ illustrating the size of a population where $\mathrm{j}$ is the position in R-dimension unique element and $\mathrm{G}$ demonstrates the generation to that the population belongs. $\mathrm{i}$ is an index for each element in $p_{G}^{i}$; these triple steps are briefly explained Engelbrecht (2007), Rahnamayan (2008), Das (2005).

\section{Mutation}

In this stage, three vectors are randomly selected so that they are mutually different. The result is a directed vector that is shown in the form of equation (18) for each vector inside the population.

$x_{j}^{(i)}=p_{j}^{\left(r_{1}\right)}+F^{*}\left(p_{j}^{\left(r_{2}\right)}-p_{j}^{\left(r_{3}\right)}\right)$

Where $i=1, \ldots, N_{p}$ and $, r_{1}, r_{2}$ and $r_{3}$ are mutually different random vectors that are selected based on $\left\{1, \ldots, N_{p}\right\}$.

\section{Recombination}

In this stage mutated vector components are transferred to candidate vector with equal probability of $C_{r}$; otherwise, equivalent component will be substituted in main vector and shown in the form of equation (19).

$$
u_{j, G}^{(i)}=\left\{\begin{array}{l}
x_{j, G}^{i} \text { if } \operatorname{rand}(j) \leq c_{r} \text { or } j=\operatorname{rand}() \\
p_{j, G}^{i} \text { else }
\end{array}\right.
$$

Where rand(j) demonstrates a $j^{\text {th }}$ call of a random function that is a number between $\{0$ and $1\}$. To make sure that at least one component is transferred to test vector, one component is transferred from the mutated vector to test vector disregarding $C_{r}$. So, for each candidate vector one component is selected for transferring to a next generation.

\section{Selection}

To select residuals, each vector is compared to corresponding candidate vector. As a result, each of them that is more competent will be transferred to the next generation.

By selecting vector $x_{G}^{(i)}$ or $P_{G}^{(i)}$ as a member of next generation $G+1$, one may write according to equation (20).

$p_{G+1}^{(i)}=\left\{\begin{array}{l}x_{G}^{(i)}, \text { if } \quad f\left(p_{G}^{(i)}\right) \leq f\left(\mathrm{x}_{G}^{(i)}\right) \\ p_{G}^{(i)} \quad \text { else }\end{array}\right.$

In the algorithm (1) DE performance would be introduced as a pseudocode.

\section{Algorithm 1. D E}


Input: Initialize and evaluate population $N_{p}$

\section{Repeat}

$$
\begin{aligned}
& \text { For } i=1 \leq N_{p} \quad d o \\
& \text { Select } p^{\left(r_{1}\right)} \text { to } p^{\left(r_{3}\right)} \text {, where } r_{1} \neq r_{2} \neq r_{3} \neq i \\
& / / \text { Selecting } \mathrm{R} \text {, as the dimension of a particle } \\
& j_{\text {rand }}=\text { floor }(R * \operatorname{rand}(0,1)) \\
& \text { For } j=1, \ldots, R \text { do } \\
& \text { if } \operatorname{rand}(0,1) \leq C_{r} \text { or } j=j_{\text {rand }} \text { then } \\
& x_{j}^{(i)}=p_{j}^{\left(r_{1}\right)}+F^{*}\left(p_{j}^{\left(r_{2}\right)}-p_{j}^{\left(r_{3}\right)}\right) \\
& \text { else } x_{j}^{(i)}=p_{j}^{(i)}
\end{aligned}
$$

end if

end for

end for

// select next generation

$$
\begin{gathered}
\text { for } i=1 \leq N_{p} \quad d o \\
\text { If } f\left(p^{(i)}\right) \leq f\left(\mathrm{x}^{(i)}\right) \text { then } \\
p^{(i)}=x^{(i)}
\end{gathered}
$$

end if

end for

\section{Termination conditions}

\section{Multiple Model Variable Rate Particle Filter with Differential Evolution}

Degeneracy phenomenon is a weakness of the particle filter. This phenomenon is resulted from variance of sample weights, and it is still a problem in MM-VRPF structure.

Many efforts have been made to generate a new group of particles that can generate higher weights such that these particles are substituted for particles with much smaller weights. In this paper, differential evolution algorithm is exploited to obtain such particles. These particles have the most proper unique values. With this regard, the fitness function in $\mathrm{DE}$ is a function for calculating the weight of a particle.

In the algorithm (2) the result of merging DE and MM-VRPF algorithm is presented. Lines starting by $(/ / /)$ demonstrate changes that are resulted after merging.

\section{Algorithm 2. MM-VRPF with DE}

Input: Initialization

Set $\mathrm{t}=0$ 
For $i=1 \leq N_{p}$, draw equally weighted samples, $x_{0}^{i} \sim p\left(x_{0}\right)$ from the predefined.

\section{Initial step}

/ / / distribution and set $\mathrm{t}=1$ with optimal states that are calculated by differential Evolution.

\section{Propagation step}

Set $k=N+t-1$

for $i=1 \leq N_{p}$

- during the neighborhood $N_{t}^{i}$ are incomplete

* Set $\mathrm{k}=\mathrm{k}+1$ and draw samples form the proposal distribution

$x_{k}^{i} \sim q\left(x_{k} \mid x_{k-1}, y_{t}\right)$ until $\tau_{k} \geq t$.

/ / / The particles are optimized by differential evolution.

/ / / Mixing particles that is achieved from two previous steps with the parameter $N_{d}$.

\section{Weight update step}

Calculate the particle weights

$$
w_{t}^{i} \propto w_{t-1}^{i} \frac{p\left(y_{t} \mid x_{N_{t}}^{i}\right) p\left(x_{N_{t-1}+1: \mathrm{N}_{t}^{+}} \mid x_{N_{t-1}^{i}}\right)}{q\left(x_{N^{+}{ }_{t-1}+1: \mathrm{N}^{+}} \mid x_{N^{+}{ }_{t-1}}^{i}, y_{0: t}\right)}
$$

Normalize the particle weights.

\section{Resampling step}

Resample $\left\{\mathrm{x}_{\mathrm{N}_{\mathrm{t}}}^{\mathrm{i}}, \mathrm{w}_{\mathrm{t}}^{\mathrm{i}}\right\}_{\mathrm{i}=1}^{\mathrm{N}_{\mathrm{P}}}$ if effective sample size $\widehat{\mathrm{N}}_{\mathrm{eff}}=\frac{1}{\sum_{\mathrm{i}=1}^{\mathrm{Np}}\left(\mathrm{w}_{\mathrm{t}}^{\mathrm{i}}\right)^{2}}$, is Below a

preset a threshold.

Set $t=t+1$.

\section{Iterate through Propagation step}

The difference between proposed mechanism and MM-VRPF algorithm is that it selects a number of particles based on differential evolution algorithm instead of applying a probability distribution. Subsequently, $N_{d}$ optimized samples are combined with other remained samples derived from the probability distribution and constitute a set of optimized samples that can be utilized in next steps of algorithm structure. In other words, samples are optimized by differential evolution algorithm. Then, they are combined with a random set obtained by probability distribution so that samples result in better response.

\section{SimULATION RESULTS}

In this section a comparison between proposed method and VRPF and MM-VRPF methods is performed. The practical application of our method in trajectory tracking of maneuvering target specially its bearing-only will be investigated. On this basis, for observation y at time t equation (21) can be written (Sanjeev, 2004). 


$$
y_{t}=\arctan \left(\frac{l_{1}-l_{10}}{l_{2}-l_{20}}\right)+v_{t}
$$

Where $\arctan ($.$) demonstrates nonlinear relationship, \mathrm{v}_{\mathrm{t}} \sim \mathrm{N}\left(0, \sigma_{\theta}^{2}\right)$ is the sensor noise and $\left[l_{10}-l_{20}\right]^{T}$ denotes the position of sensor (Sanjeev, 2004).

There are two efficiency measures for evaluation of tracking filters; time averaged root mean

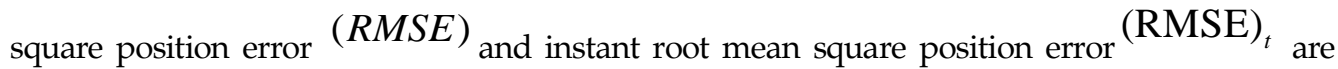
mentioned in equation (22). The achieved values for these two measures are obtained

by Monte Carlo method with L=100 runs (Ulker, 2012).

$$
\begin{aligned}
& R M S E_{t}=\sqrt{\frac{1}{T} \sum_{i=1}^{L}\left(\hat{l}_{\mathrm{t}}^{i}-l_{1 t}^{i}\right)^{2}+\left(\hat{l}_{2 t}^{i}-l_{2 t}^{i}\right)^{2}} \\
& R M S E=\sqrt{\frac{1}{L T} \sum_{t=1}^{T} \sum_{i=1}^{L}\left(\hat{l}_{\mathrm{t}}^{i}-l_{1 t}^{i}\right)^{2}+\left(\hat{l}_{2 t}^{i}-l_{2 t}^{i}\right)^{2}}
\end{aligned}
$$

Where $\mathrm{T}$ is an index to the last observation. Moreover, for executing it run $\hat{l}_{t}^{i}$ and $l_{t}^{i}$ values respectively state estimated and actual positions at time $t$.

In the figure 1.(a) desired trajectory is depicted. According to mentioned scenario in (Sanjeev, 2004) target and observer start their motion from origin with constant velocity of 4 and 5 knots, respectively and courses of -150 and 140, respectively. After that, the target executes a maneuver with constant turn rate $24^{\circ}$ /min between 20 and 25 minutes. Finally, the same course will be maintained till the end. After movement, observer experiences a maneuver in (12-16) time interval with constant turn rate of $30^{\circ} / \mathrm{min}$ to reach 20 course. For this scenario, it is assumed that the total number of observations is 40 , and the period of observation is 1 minute.

To observe the behavior of mentioned methods in a description of target motion, initial conditions are set using actual values. For instance, Gaussian value with $\left(\sigma_{\theta}=1.5\right)$ for bearing and $\left(\sigma_{r}=100 \mathrm{~m}\right)$ for range are chosen. Additionally, considering unique features such as displacement parameters and velocity of the target, the values of $\mathrm{P}$ transition matrix are selected as shown in equation (23) Ulker (2012), Sanjeev (2004).

$$
P=\left[\begin{array}{lcr}
0.5 & 0.25 & 0.25 \\
0.35 & 0.45 & 0.2 \\
0.35 & 0.2 & 0.45
\end{array}\right]
$$

Table 1 represents sojoum time distribution parameters for MM-VRPF and VRPF (Ulker, 2012). This table considers three states; $(n=1)$ for modeling direct form of motion and $(n=2,3)$ to model motion maneuvers of target. The multiple model structure can switch between these states.

In Figure 1 sections (b), (c) and (d) explain state arrival the and trajectory points generated by the VRPF, MM-VRPF and MM-VRPF with DE respectively. Figure 1.(c) and (d) show the MM- 
VRPF and MM-VRPF with DE structure are capable of locating frequent states at bearing region while using a parsimonious state representation for the smooth regions of the trajectory. Figure 2 depicts graphs resulted from tracking operation. Figure 2.(a), (b) and (c) respectively illustrate tracking operation based on VRPF, MM-VRPF and MM-VRPF with DE. It is obvious that tracking curve particularly in bearing only regions have much higher quality in MM-VRPF and MM-VRPF with DE than VRPF. Furthermore, focusing on MMVRPF with DE graph may help us to understand its relative superiority to MM-VRPF technique in path estimation.

Figure 3 shows $R M S E_{t}$ values for three mentioned structures. In figure 3.(a) the diagram of $R M S E_{t}$ versus $N_{p}=2000$ and in figure 3.(b) versus $N_{p}=4000$ are shown using 40 observations for mentioned scenarios. In addition, numerical values of RMSE for $N_{p}=$ 2000 and $N_{p}=4000$ could be seen in table 2 . Investigating values presented in table 2 together with $R M S E_{t}$ diagram in figure 3, the superiority of proposed structure could be concluded.

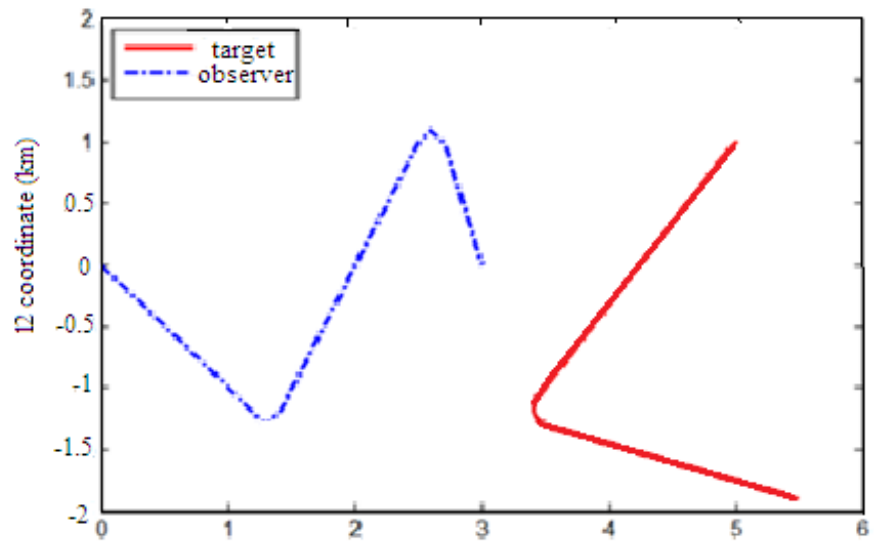

(a) 11 coordinate $(\mathrm{km})$

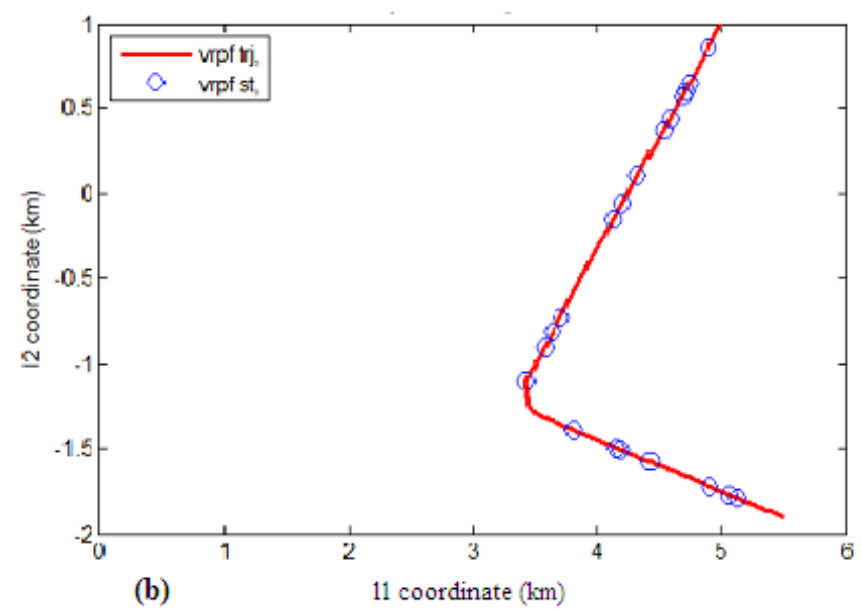


Asia Pacific Journal of Energy and Environment, Volume 2, No 2 (2015)
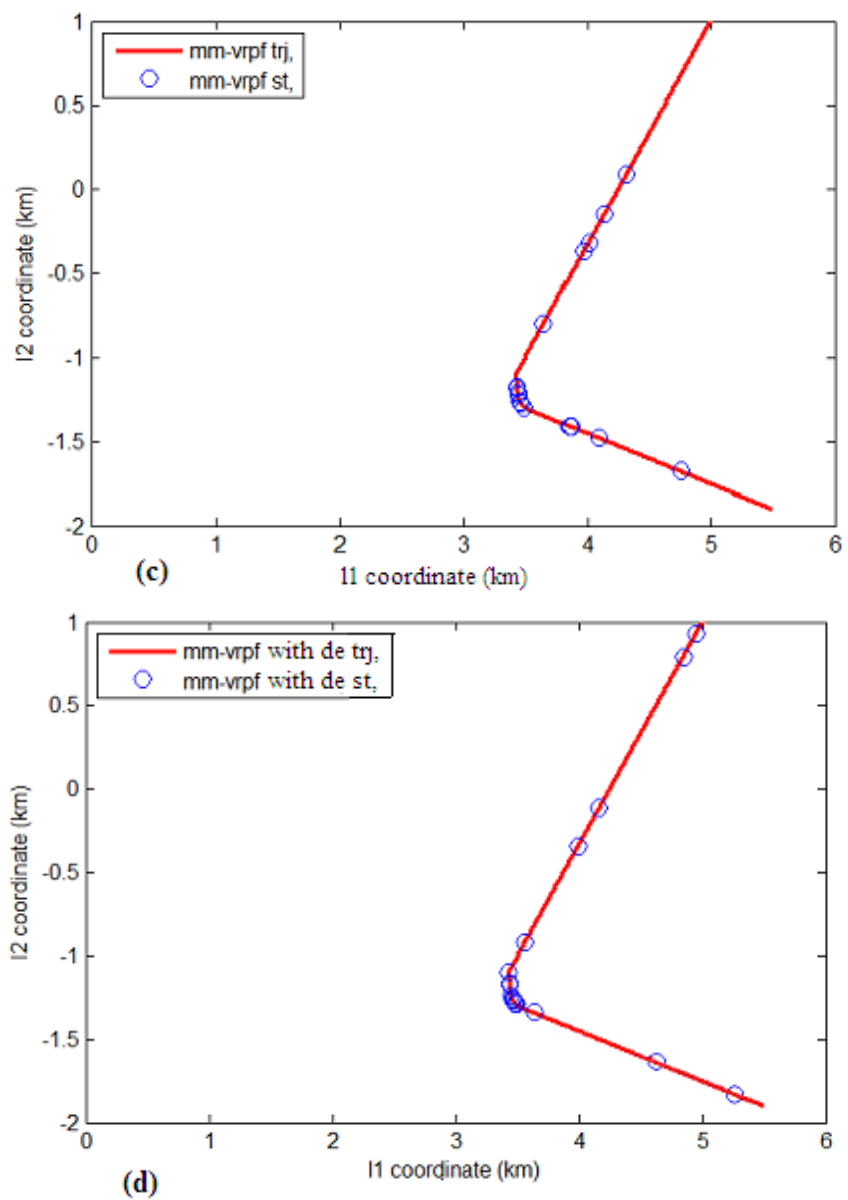

Figure 1.(a) Observer and target trajectories for desired scenario. Trajectories and states of a particle generated by (b) the VRPF, (c) the MM-VRPF and (d) the MM-VRPF with DE.

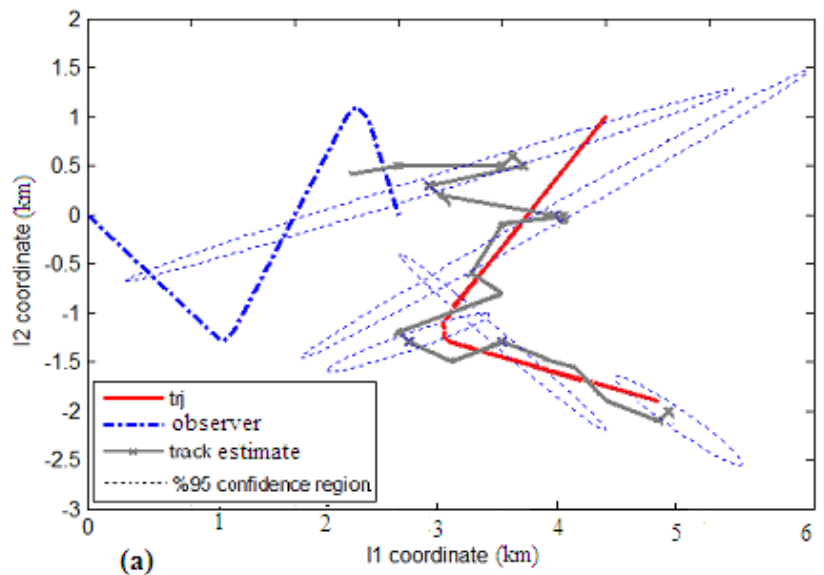


Asia Pacific Journal of Energy and Environment, Volume 2, No 2 (2015)

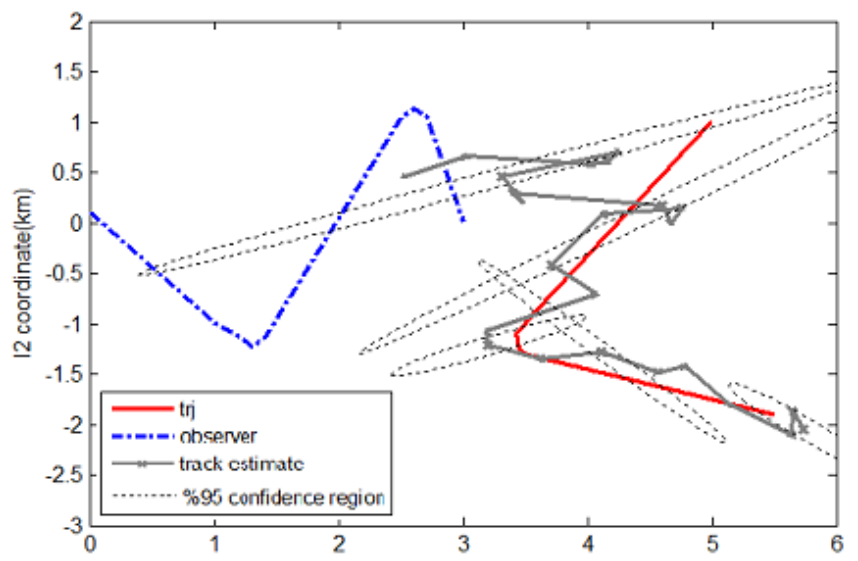

(b)

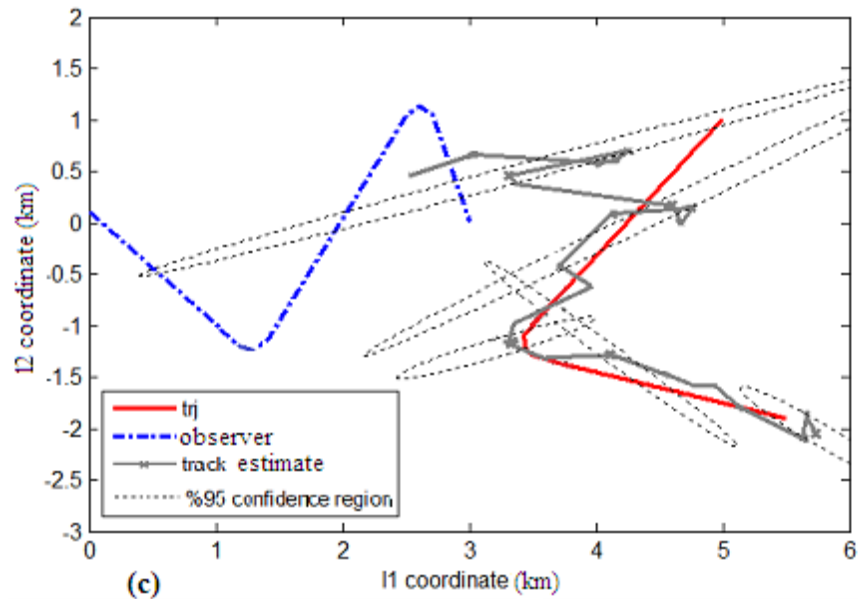

Figure 2. Tracker results by (a) the VRPF, (b) the MM-VRPF, (c) the MM-VRPF with DE.

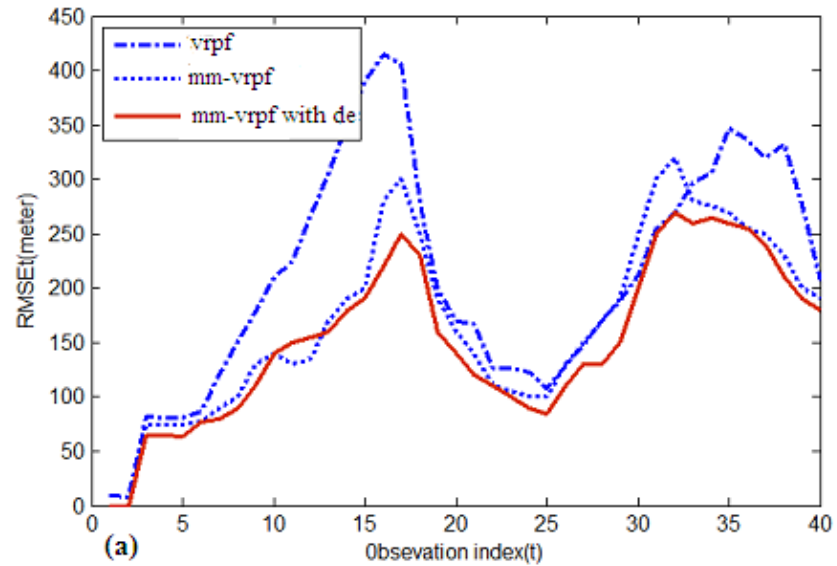




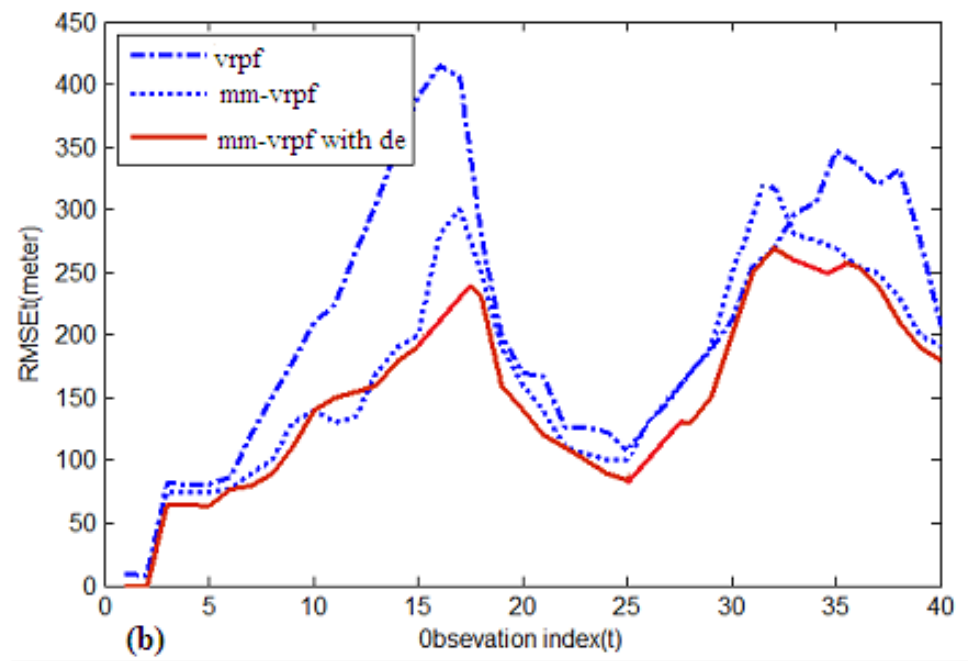

Figure 3. $R M S E_{t}$ versus time $\mathrm{t}$ for true initials by (a) $N_{p}=2000$, (b) $N_{p}=4000$.

Table 1. MM-VRPF and VRPF parameters for the desired scenario.

\begin{tabular}{|l|l|l|l|}
\hline \multirow{2}{*}{} & \multicolumn{2}{|c|}{ MM-VRP } & \multirow{2}{*}{ VRPF } \\
\cline { 2 - 3 } & MOD.1 & MOD.2,3 & \\
\hline$\mu_{T, n}, \sigma_{T, n}$ & $(0,100)$ & $(0,100)$ & $(0,100)$ \\
$\mu_{p, n}, \sigma_{p, n}$ & $(0,500)$ & $( \pm 1100,3000)$ & $(0,5000)$ \\
$\alpha_{n}, \beta_{n}$ & $(1.5,4)$ & $(0.5,0.35)$ & $(0.5,6.5)$ \\
$\tau_{n}$ & $(0)$ & $(0.5)$ & $(0)$ \\
\hline
\end{tabular}

Table 2. RMSE for varying particle size obtained by using true initials for the desired Scenario.

\begin{tabular}{|c|c|c|c|}
\hline & (VRPF) & (MM-VRPF) & (MM-VRPF with DE) \\
\hline$N_{p}=2000$ & 234.55 & 166.89 & 156.20 \\
\hline$N_{p}=4000$ & 233.44 & 149.36 & 138.50 \\
\hline
\end{tabular}

\section{CONCLUSION}

In this paper a new approach to merging DE structure with MM-VRPF structure is proposed. In this approach MM-VRPF with DE structure is suggested to improve degeneracy phenomenon that is a consequence of increase in sample weights. This method combines optimized samples generated by DE algorithm with other samples obtained from probability distribution applied to variable rate structure. As a result an optimized set of samples is achieved that will be used for estimation. The simulation results revealed relative superiority of this method in tracking high maneuver points of targets and error reduction. 


\section{REFERENCES}

Arulampalam S, Maskell S, Gordon N and Clapp T. A Tutorialon Particle Filters for Online Nonlinear/Non-gaussian Bayesian Tracking. IEEE Trans. Signal Process. 2002: 174-188.

Bloomer L, Gray JE. Are more models better?: The effect of the model transition matrix on the IMM filter. In: 4th Southeastern Symp. System Theory; 2002; Huntsville, CANADA. pp. 20-25.

Boers Y. On the Number of Samples to be Drawn in Particle Filtering. IEEE Colloquium on Target Tracking: Algorithms and Applications. 1999: 5/1- 5/6.

Das S, Konar A, Chakraborty U. Improved differential evolution algorithms for handling noisy optimization problems. IEEE Congress on Evolutionary Computation. 2005: 1691-1698.

Doucand R, Capp_e O. Comparison of resampling schemes for particle Filtering. In: Image and Signal Processing and Analysis IEEE 2005 Proceedings of the 4th International Symposium; 2005; USA, IEEE. pp. 64-69.

Doucet A, Godsill SJ, Andrieu C. On sequential Monte Carlo sampling methods for Bayesian filtering. Statistics and computing. 2000: 197-208.

Engelbrecht AP. Computational Intelligence An Introduction 2th Ed.New Jersy. USA: John Wiley and Sons John. 2007.

Fox D. KLD-Sampling: Adaptive Particle Filters. Int. J. Robotics Research. 2003: 985-1003.

Godsill SJ, Vermaak J, Ng W, Li JF. Models and Algorithms for Tracking of Maneuvering Objects Using Variable Rate. proc. IEEE. 2007: 1-28.

Gordon N, Salmond D, Smith AFM. Novel Approach to Non-linear and Non-Gaussian Bayesian State Estimation. Radar and Signal Processing. IEE Proceedings F. Vol. 140. No. 2. 1993: 107-113.

Gordon NJ, Salmond DJ, and Smith AFM. Novel approach to nonlinear/non-Gaussian Bayesian state estimation. in IEE Proceedings. vol. 140. 1993: 107-113.

Jilkov VP, Li XR. Online bayesian estimation of transition probabilities for markovian jump systems. IEEE Trans. Signal Process. 2004: 1620-1630.

Li X, Jilkov V. Survey of maneuvering target tracking. Part I: Dynamic models. IEEE Transactions on Aerospace and Electronic Systems, vol. 39. 2003: 1333-1364.

Punch P, Godsill SJ. Dynamical models for tracking with the variable rate particle filter. In: IEEE 15th International Conference on information fusion; 2012; USA; IEEE. pp. 1769 - 1775.

Rahnamayan S, Tizhoosh HR, Salama MMA. Opposition-based differential evolution, IEEE Transactions on Evolutionary Computation. 2008: 64-79.

Ristic B, Arulampalam S, Gordon N. In Beyond Kalman Filter:Particle Filters TrackingApplication. Artech House Publishers. Boston,Ch. 1-3. 2004: 3-65.

Sanjeev Arulampalam M, Ristic B, Gordon N, Mansell T. Bearings-Only Tracking of Manoeuvring Targets Using Particle Filters. EURASIP Journal on Signal Processing. 2004: 2351-2365.

Simon D. Optimal State Estimation Kalman, Haand Non-linear Approaches. New Jersey, USA: John Wiley and Sons, 2006.

Ulker Y, Gunsel B. Multiple model target tracking with variable rate particle filters. Elsevier Journal on Signal Processing. 2012: 417-429. 